Recall that, by Theorem $3.1, E(\mathfrak{Q}) \supset \Delta(A)$. From our definitions $\Delta(A)$ is dense in 3 so that also $E(\mathfrak{Q}) \supset \mathfrak{Z}$. Since the reverse inequality is clear, the proof is complete.

\title{
REFERENCES
}

1. I. M. Gel'fand, D. A. Raikov and G. E. Silov, Commutative normed rings, Uspehi Mat. Nauk 1 (1946), 48-146; Amer. Math. Soc. Transl. (2) 5 (1957), 115-220.

2. C. E. Rickart, General theory of Banach algebras, Van Nostrand, New York, 1960.

3. G. E. Silov, On the extension of maximal ideals, Dokl. Akad. Nauk SSSR 29 (1940), 83-84.

UNIVERSITY OF OREGON

\section{SEMI-HOMOGENEOUS FUNCTIONS}

LOUIS V. QUINTAS AND FRED SUPNICK

1. Introduction and statement of results. A function $f$ is called homogeneous of degree $n$ with respect to the set $A$, or briefly semihomogeneous if

$$
f(a x)=a^{n} f(x)
$$

is satisfied for all $x$ in the domain of $f$ and all $a$ in $A$.

With each admissible $A$ there is associated a class of solutions of (1.0). E.g., let $R$ denote the set of all real numbers and let $f$ be a function on $R$ to $R$. If $A$ consists only of the irrationals, then $f(x)$ $=c x^{n}(c=f(1))$ is the unique solution of (1.0). On the other hand, if $A$ consists only of the rationals, then in addition to $f(x)=c x^{n},(1.0)$ has other solutions (e.g., if $n$ is any nonzero integer and $f(x)=x^{n}$ or 0 accordingly as $x$ is rational or irrational).

We are interested in studying decompositions of the set of admissible $A$ 's into classes and in characterizing the solutions of (1.0) corresponding to these classes. In this paper we show how this can be done in a natural way for semi-homogeneous functions of a real variable. We note that in this case our methods apply to

$$
f(a x)=p(a) f(x) \quad(a \in A \subset R),
$$

where $p$ is a product-preserving function on $R$ to $R$ (cf. [1]). We shall therefore confine our attention to (1.1).

Received by the editors March 8, 1962. 
Let $C(p, A)$ denote the set of all functions on $R$ to $R$ satisfying (1.1). Let $R^{*}=R-\{0\}$ and $A^{*}$ be the multiplicative subgroup of $R^{*}$ generated by $A-\{0\}$. It is easy to see that if $A$ contains a set of generators for $R^{*}$, then $f(x)=f(1) p(x)$. Thus, the problem of determining which subsets of $R$ lead to the solution $f(x)=f(1) p(x)$ of (1.1) is equivalent to classifying those subsets of $R$ which generate $R^{*}$ (cf. §5). With respect to the general case we first establish the following lemma.

Lemma. If $p \not \equiv 0$ and $p \not \equiv 1$ on $A^{*}$, then $C(p, A)=C\left(p, A^{*}\right)(c f . \S 2)$.

Thus we are led to consider the problem: For an arbitrary subgroup $A^{*}$ of $R^{*}$, how can the members of $C\left(p, A^{*}\right)$ be characterized?

The general answer to the above question is given in Theorem 1 . Let $R^{*} / A^{*}$ denote the multiplicative quotient group of $R^{*} \operatorname{modulo} A^{*}$, $B$ a set of representatives for $R^{*} / A^{*}$, and $p$ a product-preserving function on $R$ to $R$ such that $p \not \equiv 0$ and $p \not \equiv 1$ on $A^{*}$ (cf. [2]).

Theorem 1. (i) $f \in C\left(p, A^{*}\right)$ if and only if

$$
f(x)=\left\{\begin{array}{cl}
F(b) p(x) & x=a b, a \in A^{*}, b \in B, \\
0 & x=0,
\end{array}\right.
$$

where $F$ is a function on $B$ to $R$.

(ii) If $f \in C\left(p, A^{*}\right)$, then $f$ is uniquely determined when it is known on $B(c f . \S 2)$.

An explicit classification and description of the solutions of (1.1) depends on a consideration of the different possibilities for $A^{*}$ and $p$. We note that $A^{*}$ is either dense in the positive reals, dense in $R$, or is discrete and countable. Also, if $p$ is continuous on $R^{*}(p \neq 0)$, then $p$ is of the form $p(x)=|x|^{k}$, or $p(x) \in\left\{x^{k}(0<x),-|x|^{k}(x<0)\right\}$ where $k$ is a real constant.

Theorem 2. Let $A^{*} \subset R^{*}, p \neq \equiv, 1$ on $A^{*}$, and $f \in C\left(p, A^{*}\right)$. If $p$ is continuous on $R$ and $A^{*}$ is dense in $R$, then $f$ is of type

$T_{1}: f(x)=c p(x)$, "c" a constant,

$T_{2}: f$ is continuous only at $x=0$, and the closure $\mathrm{Cl}(G(f))$ of its (cartesian) graph $G(f)$ (in $\left.E_{2}\right)$ is a set of curves $\{y=c p(x)\}$ with $\{c\}$ bounded, or

$T_{3}: f$ is totally discontinuous and $\mathrm{Cl}(G(f))$ is a set of curves $\{y=c p(x)\}$ with $\{c\}$ unbounded. $\mathrm{Cl}(G(f))$ may be the entire plane if $f$ is of type $T_{3}(c f . \S 3)$.

REMARK. It is of interest to note that if $f$ is a function which satis- 
fies $f(x+y)=f(x)+f(y)$, then the classic description of $f$ given by G. Hamil (namely, that $f$ is of the form $f(x)=c x$, or is totally discontinuous and the graph of $f$ is dense in the entire plane) is contained within our Theorem 2 (cf. [3]). This follows from the fact that $f(x+y)=f(x)+f(y)$ implies $f$ is homogeneous of degree 1 with respect to the rationals, i.e., $f(a x)=a f(x)$ for all rational numbers $a$.

Let $A^{*}$ be an infinite discrete subgroup of $R^{*}$. Let $g$ denote the smallest member of $A^{*}$ such that $1<g, G_{0} \equiv\{(x, f(x)): 1 \leqq x<g\}$ where $f$ is a function on $R$ to $R, D_{a}$ the transformation of $E_{2}$ into $E_{2}$ defined by $D_{a}(x, y)=(a x, p(a) y)(a \in R)$, and $D_{a} G_{0}$ the image of $G_{0}$ under $D_{a}$.

THeOREM 3. If $A^{*}$ is an infinite discrete subgroup of $R^{*}$ containing at least one negative member and $f \in C\left(p, A^{*}\right)$, then $\left\{D_{a} G_{0}: a \in A^{*} \cup\{0\}\right\}$ is a decomposition of $G(f)$ (cf. [4] and \$4).

In Theorems 2 and 3 we have considered the cases (i) $p$ continuous and $A^{*}$ dense, and (ii) $p$ arbitrary and $A^{*}$ discrete. We note that if $p$ is discontinuous on $R^{*}$, then $p$ is totally discontinuous and the graph of $p$ is dense in the first and second or the first and third quadrants depending on whether $p(-1)=1$ or -1 , respectively. The problem of obtaining an esthetic classification of the solutions of (1.1) for the case $p$ discontinuous and $A^{*}$ dense in $R$ remains open.

\section{Proof of the Lemma and Theorem 1.}

Proof of the Lemma. Since $A-\{0\} \subset A^{*}, C\left(p, A^{*}\right)$ $\subset C(p, A-\{0\})$. If $0 \notin A$, then $C\left(p, A^{*}\right) \subset C(p, A)$. If $0 \in A$, then since $p(0)=0$ every member of $C(p, A)$ must assign 0 to 0 . But $p \not \equiv 1$ on $A^{*}$ implies there exist $a, a^{\prime} \in A^{*}$ such that $p(a) \neq p\left(a^{\prime}\right)$. Thus, $f(0)\left(p(a)-p\left(a^{\prime}\right)\right)=0$ yields $f(0)=0$ whenever $f \in C\left(p, A^{*}\right)$. Therefore, $C\left(p, A^{*}\right) \subset C(p, A)$.

Let $f \in C(p, A)$. To prove $C(p, A) \subset C\left(p, A^{*}\right)$ we use the fact that every member of $A^{*}$ is a finite product of members of $A-\{0\}$ or their reciprocals. Thus, if $s \in A^{*}$, then $s=a t$ or $s=a^{-1} t$ with $a \in A-\{0\}$ and $t \in A^{*}$. If $s=a t$, then

$$
f(s x)=f(a t x)=p(a) f(t x) .
$$

If $s=a^{-1} t$, then

$$
f(s x)=p\left(a^{-1} a\right) f\left(a^{-1} t x\right)=p\left(a^{-1}\right) f(t x) .
$$

Applying operations of the form (2.1) or (2.2) successively we obtain $f(s x)=p(s) f(x)\left(s \in A^{*}\right)$. Thus, $C(p, A)=C\left(p, A^{*}\right)$.

Proof of Theorem 1 (i). Since $R^{*} / A^{*}$ defines a partition of $R^{*}$, 
each nonzero real number $x$ has a unique representation in terms of $A^{*}$ and $B$. Namely, $x=a b\left(a \in A^{*}, b \in B\right)$. Thus (1.2) defines a function on $R$ to $R$.

Let $f \in C\left(p, A^{*}\right)$. Since $p \not \equiv 0$, then $p(b) \neq 0\left(b \in R^{*}\right)$. Thus $f(x)$ $=p(x)(p(b))^{-1} f(b)$ whenever $x=a b\left(a \in A^{*}, b \in B\right)$. Since $p \neq \equiv 1$ on $A^{*}$, then $f(0)=0$. Consequently, $f$ can be expressed in the form (1.2) with $F$ the function on $B$ to $R$ defined by $F(b)=(p(b))^{-1} f(b)$.

Conversely, let $f$ be defined by (1.2). If $s \in A^{*}$ and $x \in R^{*}$, then $x=a b$ and $s x=s a b\left(a \in A^{*}, s a \in A^{*}, b \in B\right)$. Thus,

$$
f(s x)=F(b) p(s x)=p(s) F(b) p(x)=p(s) f(x) .
$$

If $x=0$, then $f(s 0)=0=p(s) f(0)$. Therefore, $f \in C\left(p, A^{*}\right)$.

Proof of Theorem 1 (ii). If $f, f^{\prime} \in C\left(p, A^{*}\right)$ and $f(b)=f^{\prime}(b)(b \in B)$, then

$$
f(x)=p(a) f(b)=p(a) f^{\prime}(b)=f^{\prime}(x)
$$

whenever $x=a b\left(a \in A^{*}, b \in B\right)$, and $f(0)=0=f^{\prime}(0)$.

3. Proof of Theorem 2. By Theorem 1 the image under $f$ of each coset of $R^{*} / A^{*}$ lies on a curve, $y=c p(x)$. Specifically, if $x \in A^{*} b$, then $(x, f(x))$ lies on $y=F(b) p(x)$. Since each coset of $R^{*} / A^{*}$ is dense in $R$, the image $I$ of each coset is dense in the curve containing $I$. Each $y=c p(x)$ is continuous, hence the closure of the graph of $f$ is a set of curves $K \equiv\{y=c p(x)\}$ through the origin. If all the cosets of $R^{*} / A^{*}$ are mapped onto the same curve, then $f$ is a function of type $T_{1}$. If this is not the case, then $f$ is a function of type $T_{2}$ or $T_{3}$ accordingly as the set of coefficients $C \equiv\{c\}$ appearing in $K$ is bounded or unbounded. If $K$ contains at least two curves, it is clear that $f$ cannot be continuous at nonzero values of $x$. Suppose $C$ is bounded. If $c_{1}=\operatorname{lub} C, c_{2}=\operatorname{glb} C$, and $c_{0}=\max \left\{\left|c_{1}\right|,\left|c_{2}\right|\right\}$, then $f(x)$ lies between $-c_{0} p(x)$ and $c_{0} p(x)$ for all $x$. If $\left\{x_{i}\right\}$ is any sequence of real numbers converging to 0 , then $\lim _{x_{i} \rightarrow 0}\left\{c_{0} p\left(x_{i}\right)\right\}=0$ implies $\lim _{x_{i} \rightarrow 0}\left\{f\left(x_{i}\right)\right\}=0$. Since $f(0)=0$, we have $f$ is continuous at $x=0$. Hence, $f$ is of type $T_{\mathbf{2}}$. If $C$ is unbounded, then $f$ is unbounded in every neighborhood of the origin. Since $x=0$ is the only possible point of continuity and $f(0)=0$, $f$ is totally discontinuous and is thus of type $T_{3}$.

If the index of $A^{*}$ in $R^{*}$ is $2^{N_{0}}$ and $B$ is a set of representatives for $R^{*} / A^{*}$, then there exists a 1-1 function $F$ on $B$ to $R$. The function $f$ defined by $f(x)=F(b) p(x)\left(x=a b, a \in A^{*}, b \in B\right)$ and $f(0)=0$ is a type $T_{3}$ member of $C\left(p, A^{*}\right)$ and $G(f)$ is dense in $K \equiv\{y=F(b) p(x)$ : $b \in B\}$. But $K \equiv\{y=r p(x): r \in R\}$ is the entire plane with the exception of the points on the $y$-axis having nonzero ordinates. Hence $\mathrm{Cl}(G(f))$ is the entire plane. This completes the proof of Theorem 2 . 
4. Proof of Theorem 3. Since $A^{*}$ is discrete and not finite there exists a smallest member $g \in A^{*}$ such that $1<g$ and $B=\{x \in R$ : $1 \leqq x<g\}$ is a set of representatives for $R^{*} / A^{*}$.

$A^{*}$ induces the following decomposition of $R^{*}$. The set of positive reals is decomposed into intervals $g^{n} \leqq x<g^{n+1}$ where $n$ ranges over the integers. $A^{*}$ also contains a largest member $h$ such that $h \leqq-1$. We now decompose the set of negative reals into the sets $h g^{n+1}<x$ $\leqq h g^{n}$. In this way $R^{*}$ is decomposed into intervals each of which is the image of the set $B$ under multiplication by a suitable member of $A^{*}$. This decomposition of $R^{*}$ induces a decomposition of $G(f)$ into the following sets:

$$
G_{n}=\left\{(x, f(x)): g^{n} \leqq x<g^{n+1}\right\}, \quad G_{n}^{\prime}=\left\{(x, f(x)): h g^{n+1}<x \leqq h g^{n}\right\},
$$

and $\{(0,0)\}$, where $g$ and $h$ are as described above and $n$ ranges over the integers.

We note that $G_{0}$ is the graph of $f$ in $E_{2}$ which corresponds to $B$. Since $f$ is completely determined when we know its values on $B$, we seek a relation between the set $G_{0}$ and the other sets of this decomposition of $G(f)$. Applying $D_{0^{n}}$ to $G_{0}$ we obtain

$$
\begin{aligned}
\left\{\left(g^{n} x, p\left(g^{n}\right) f(x)\right): 1 \leqq x<g\right\} & =\left\{\left(g^{n} x, f\left(g^{n} x\right)\right): 1 \leqq x<g\right\} \\
& =\left\{(y, f(y)): g^{n} \leqq y<g^{n+1}\right\} .
\end{aligned}
$$

Therefore, $D_{g^{n}} G_{0}=G_{n}$. Similarly, $D_{h g^{n}} G_{0}=G_{n}{ }^{\prime}$ and $D_{0} G_{0}=\{(0,0)\}$.

$G(f)$ thus decomposes into sets each of which is the image of the set $G_{0}$ under a transformation $D_{a}$. Therefore, $\left\{D_{a} G_{0}: a \in A * \cup\{0\}\right\}$ is a decomposition of $G(f)$. This completes the proof of Theorem 3 .

Theorems analogous to Theorems 2 and 3 may be obtained when $A^{*}$ is a subgroup of the positive reals or when $p$ is continuous only on $R^{*}$.

5. Remarks on the solution $f(1) p(x)$. Let $A \subset R$ and $\mu$ denote the Lebesgue measure on $R$.

1. If $\mu(A)>0$ and $A$ contains at least one negative member, then $f(1) p(x)$ is the unique solution of (1.1).

Proof. Let $R_{p}^{*}$ denote the positive reals. If $A_{p}=A^{*} \cap R_{p}^{*}$, then $\mu\left(A_{p}\right)>0$. Since $\mu\left(\ln \left[A_{p}\right]\right)>0$, we have

$$
D\left(\ln \left[A_{p}\right]\right) \equiv\left\{x-y: x=\ln (a), y=\ln (b) a, b \in A_{p}\right\}
$$

contains an open interval containing the origin (cf. [5]). Thus,

$$
e^{D\left(\ln \left[A_{p}\right]\right)}=\left\{e^{\ln (a)-\ln (b)}=a / b: a, b_{\leftarrow}^{*} \in A_{p}\right\}
$$

contains an open interval containing 1 . Since every positive real 
number has an $n$th root in this interval, $e^{D\left(\ln \left[A_{p}\right]\right)}$ generates $R_{p}^{*}$. Hence, $R_{p}^{*} \subset A^{*}$. Since $A$ contains a negative number $-1 \in A^{*}$. Therefore, $A^{*}=R^{*}$ and the assertion follows from the Lemma.

2. There exist sets $A \subset R$ such that $\mu(A)=0$ and $f(1) p(x)$ is the unique solution of (1.1)

Proof. Let $C$ denote the Cantor set and $A=\left\{e^{c}: c \in C\right\} \cup\{-1\}$. It is a theorem of Steinhaus that for every number $d$ between 0 and 1 there are two numbers of $C$ which differ by $d$ (cf. [6]). Thus, $\left\{e^{c}: c \in C\right\}$ generates $R_{p}^{*}$. Hence, $A^{*}=R^{*}$.

3. It is clear that if $A$ is countable then $A^{*} \neq R^{*}$. We also note that $R^{*}$ does not contain any minimal set of generators.

\section{Notes AND REFERENCES}

1. The special case $p(a)=a(a \in A \subset R)$ was first considered by the authors and the results announced in Abstract 577-8, Notices Amer. Math. Soc. 8 (1961), 51.

2. If $0 \in A$ and $p \equiv 1$, then $C(1, A)$ is the set of all constant functions. If $0 \notin A$, $A$ is not null, and $p \equiv 1$ on $A^{*}$, then $C(1, A)$ is the set of all functions which are constant on the cosets of $R^{*} / A^{*}$ and $f(0)$ is an arbitrary constant.

3. G. Hamil, Eine Basis aller Zahlen und die unstetige Lösungen der Funktionalgleichung $f(x+y)=f(x)+f(y)$, Math. Ann. 60 (1905), 459-462.

4. By a decomposition of a set $X$ we mean a disjoint family of subsets of $X$ whose union is $X$.

5. P. R. Halmos, Measure theory, Van Nostrand, Princeton, N. J., 1950, Theorem B, p. 68.

6. H. Steinhaus, A new property of G. Cantor's set, Wektor 7 (1917). (Polish) See also, J. F. Randolph, Distances between points of the Cantor set, Amer. Math. Monthly 47 (1940), 549.

City College 